\title{
N91-13311.
}

\section{Mutual Coupling between Circular Apertures on an Infinite Conducting Ground Plane and Radiating into a Finite Width Slab}

\author{
by \\ Christos Christodoulou \\ Associate Professor \\ Electrical Engineering Department \\ University of Central Florida \\ Orlando, FL. 32816
}

The problem of electromagnetic coupling between two horns is of interest for the Microwave Reflectometer Ionization Sensor (MRIS) that will be used in the Aeroassist Flight Experiment (AFE). Laboratory measurements of mutual coupling betwcen conical horns (using a flat metallic reflector to simulate a critically dense plasma outside) have sliown a strong dependence on the finite dimesnions of the shuttle tile over the apertures. Since both, the dielectric tile and the plasma outside the tile reflect microwaves, a study should be done to isolate the two mechanisms so that the MRIS rentry flight data can be interpreted correctly. Once the coupling due to the tile itself is determined then the location of the critical electron number density layers can be determined.

As a first attempt to tackle this problem the Gcometrical Theory of Diffraction was used to "modify" the existing solution [1] to mutual coupling betwcen apertures with infinite dielectric shects. Figure 1 depicts the main rays that contibure to coupling betwen the two horns.

The mutual adnittance for two apertures in a infinte ground plane and racliating into a finite width dielectric slab can be written as [2]:

$$
Y_{12}=Y_{12}^{o}+\sum_{n=1}^{N} Y_{12}^{n}
$$

where $Y_{12}^{\circ}=$ mutual inductance for apertures in ground pline,$N=$ the number of reflected rays, and

$$
Y_{12}^{-n}=\frac{1}{V_{1} V_{2}} \int_{0}^{2 \pi} \int_{0}^{a}\left[E_{\rho}^{1}(\rho, \phi) H_{\phi}^{r, l}(\rho, \phi)-E_{\phi}^{1}(\rho, \phi) H_{,, d}^{r, d}(\rho, \phi)\right] \rho d \rho d \phi
$$

By using the equivalent current method, aperture theory to determine the radiated fields inside the dielectric tile, and ray tracing the following contrilintions to mutual coupling were determined :

\section{Coupling due to Reflection}

$$
\begin{aligned}
Y_{12}^{n}= & {\left[\frac{j(2 / \pi) R 1 k_{d} a \cos ^{2} \theta_{0}}{J_{1}^{2}\left(x_{11}\right)\left(x_{11}^{2}-1\right) Z_{d t} s_{01}} \frac{J_{1}^{\prime}\left(k_{d} a \sin \theta_{0}\right)}{\left[1-\left(\frac{k_{d} a \sin \theta_{0}}{x_{11}}\right)^{2}\right]}\right.} \\
& \sqrt{\frac{s_{01}}{s_{02}\left(s_{02}+s_{01}\right)}} \sqrt{\left.\frac{s_{02}}{s_{03}\left(s_{03}+s_{02}\right)} e^{-j k_{d}\left(s_{01}+s_{02}+s_{03}\right)}\right]} \\
& \int_{0}^{2 \pi} \int_{0}^{a}\left[J_{1}^{\prime}\left(x_{11} \rho / a\right) \cos ^{2} \phi\left(x_{11} / a\right) e^{-j k_{d} \rho c \operatorname{sis} \phi \sin \theta_{0}}-\right. \\
& \left.\frac{J_{1}\left(x_{11} \rho / a\right)}{\rho} \sin ^{2} \phi c^{-j k_{d} \rho \cos \phi \sin \theta_{0}}\right] \rho d \rho d \phi
\end{aligned}
$$


where $R 1$ is the reflection coefficient and a the aperture radius.

\section{Coupling due to Diffraction from Bottom Wedges}

$$
Y_{12}^{n}=-\left(4 j / Z_{d}\right)\left(k_{d}\right)^{1 / 2} D_{h} \frac{1}{k_{d} s_{o}}\left(\frac{s_{o}^{\prime}}{s_{o}}\right)^{1 / 2}\left(\frac{J_{1}^{2}\left(k_{d}(a) s i n^{2}\left(\phi_{0}^{\prime}\right)\right.}{\left(x_{11}^{2}-1\right)\left[k_{d}\left(s_{o}^{\prime}+s_{o}\right)\right]^{1 / 2}}\right) e^{-j k_{d}\left(s_{o}^{\prime}+s_{\nu}\right)}
$$

where $D_{h}$ is the diffraction coefficient.

\section{Coupling due to Diffraction from Top Wedges}

$$
\begin{aligned}
Y_{12}^{n} & =B_{1} \frac{2 J_{1}\left(x_{11}\right) J_{1}\left(k_{d} a \sin \theta_{o}\right)}{\left(x_{11} / a\right)\left(k_{d} \sin \theta_{o}\right)} \\
& +B_{3} \frac{\left(k_{d} \sin \theta_{0} a\right) J_{2}\left(x_{11}\right) J_{1}\left(k_{d} \sin \theta_{0} a\right)-x_{11} J_{1}\left(x_{11}\right)}{\left(x_{11} / a\right)^{2}-\left(k_{d} \sin \theta_{0}\right)^{2}} \frac{J_{2}\left(k_{d} \sin \theta_{0} a\right)}{\left(x_{11} / a\right)^{2}-\left(k_{d} \sin \theta_{0}\right)^{2}} \\
& +B_{2} \frac{\left(k_{d} \sin \theta_{0} a\right) J_{0}\left(x_{11}\right) J_{-1}\left(k_{d} \sin \theta_{0} a\right)-x_{11} J_{-1}\left(x_{11}\right) J_{0}\left(k_{d} \sin \theta_{0} a\right)}{\left(x_{11} / a\right)^{2}-\left(k_{d} \sin \theta_{0}\right)^{2}} \\
& +.5 B_{2}\left(\cos 2 \phi_{o}-\sin 2 \phi_{o}\right) \frac{\left(k_{d} \sin \theta_{0} a\right) J_{2}\left(x_{11}\right) J_{1}\left(k_{d} \sin \theta_{0} a\right)-x_{11} J_{1}\left(x_{11}\right) J_{2}\left(k_{d} \sin \theta_{0} a\right)}{\left(\sin 2 \phi_{o}\right) \cdot \int_{0}^{a} J_{0}\left(x_{11} \rho / a\right) J_{2}\left(k_{d d} \sin \theta_{0} \rho\right) \rho d \rho} \\
& +.5 B_{2}\left(\cos 2 \phi_{0}+\sin \right)
\end{aligned}
$$

where $B_{1}, B_{2}, B_{3}$ are constants containing information about the incident ficlds and their phase.

The contribution $Y_{11}^{n}$ to the self-admittance of one aperture due to diffraction is obtained by setting $\phi_{o}=\pi / 2$ in the above cquations.

Results from two cases with different tile thicknesses have indicated that the main contribution to mutual coupling is due to diffraction from the bottom and top (bark and front) wedges.

\section{References}

1. M.C. Bailey :" Near Field Coupling Between Elements of a Finite Planar Array of Circular Apertures", Ph.D. Dissertation, VPI\&SU, December 1972.

2. M.C. Bailey : "Mutual Coupling Between Circular Waveguide-Fed Apertures in a Rectangular Ground Plane", IEEE Trans. on Antennas and Propagation, July 1974, pp. 597-599. 


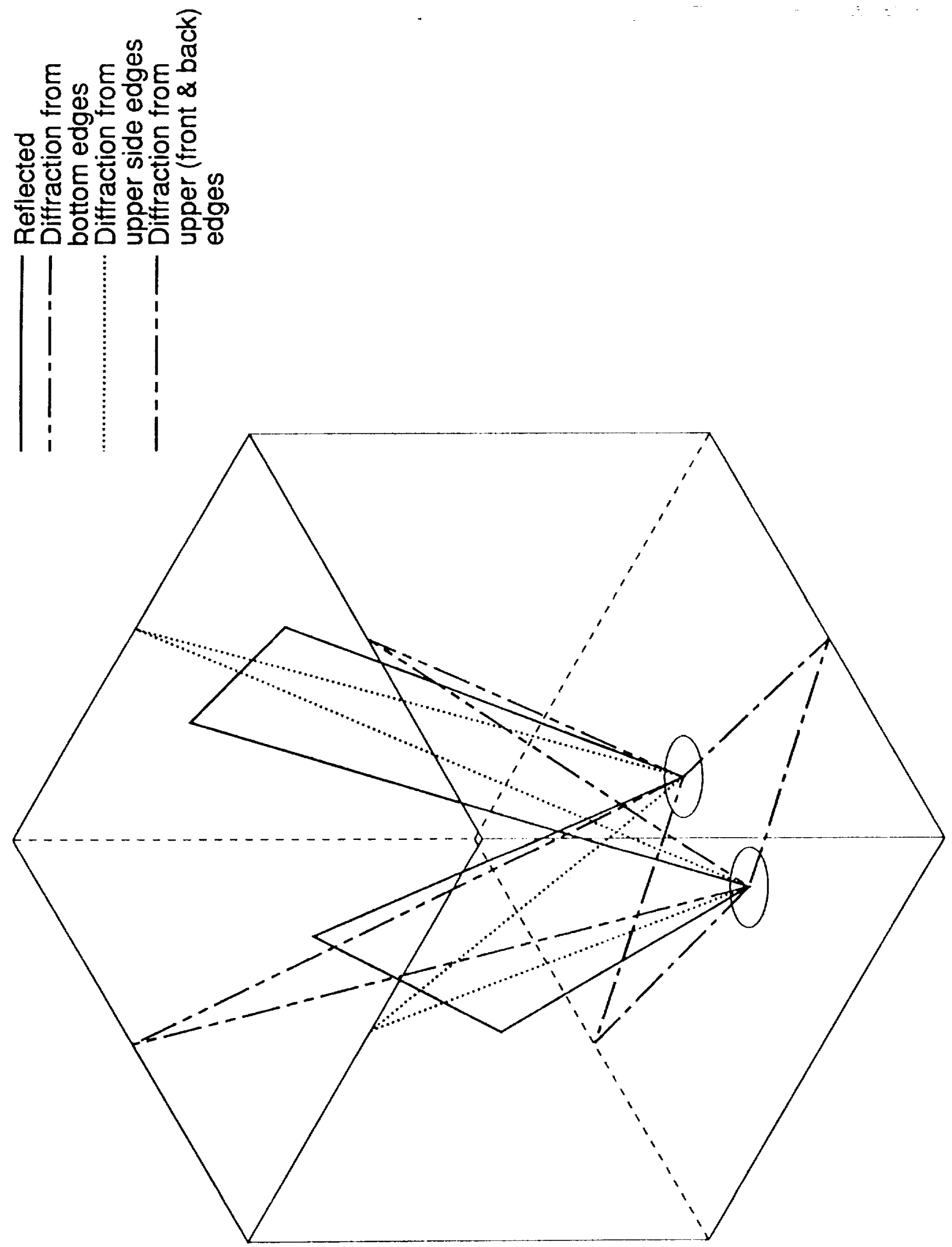

